\title{
Ab initio calculations of the lattice parameter and elastic stiffness coefficients of bcc Fe with solutes
}

\author{
Michael R. Fellinger ${ }^{\mathrm{a}, *}$, Louis G. Hector Jr. ${ }^{\mathrm{b}}$, Dallas R. Trinkle ${ }^{\mathrm{a}}$ \\ ${ }^{a}$ Department of Materials Science and Engineering, University of Illinois at \\ Urbana-Champaign, Urbana, Illinois 61801, USA \\ ${ }^{b}$ General Motors RछD Center, 30500 Mound Road, Warren, MI 48090, USA
}

\begin{abstract}
We present an efficient methodology for computing solute-induced changes in lattice parameters and elastic stiffness coefficients $C_{i j}$ of single crystals using density functional theory. We introduce a solute strain misfit tensor that quantifies how solutes change lattice parameters due to the stress they induce in the host crystal. Solutes modify the elastic stiffness coefficients through volumetric changes and by altering chemical bonds. We compute each of these contributions to the elastic stiffness coefficients separately, and verify that their sum agrees with changes in the elastic stiffness coefficients computed directly using fully optimized supercells containing solutes. Computing the two elastic stiffness contributions separately is more computationally efficient and provides more information on solute effects than the direct calculations. We compute the solute dependence of polycrystalline averaged shear and Young's moduli from the solute dependence of the single-crystal $C_{i j}$. We apply this methodology to substitutional Al, B, Cu, Mn, Si solutes and octahedral interstitial C and N solutes in bcc Fe. Comparison with experimental data indicates that our approach accurately predicts solute-induced changes in the lattice parameter and elastic coefficients. The computed data can be used to quantify solute-induced changes in mechanical properties such as strength and ductility, and can be incorporated into mesoscale models to improve their predictive capabilities.
\end{abstract}

\footnotetext{
${ }^{*}$ Corresponding author

Email address: mfelling@illinois.edu (Michael R. Fellinger)
}

Preprint submitted to Journal of Computational Materials Science

September 17, 2016

(C) 2016. This manuscript version is made available under the Elsevier user license http://www.elsevier.com/open-access/userlicense/1.0/ 
Keywords: lattice parameters, elastic constants, solutes, iron, steel, ferrite, ab initio

\section{Introduction}

Solutes strongly influence the structural and mechanical properties of steel alloys [1, 2, and steel chemistries are tuned to balance strength, hardness, toughness, and ductility for diverse applications. Small additions of solutes modify structural parameters [3, 4, 5], elastic stiffness coefficients $C_{i j}$ [6, 7, 8, 9], stacking fault energies [10, 11], dislocation cores [12], and phase stability [13, 14, 15], leading to alloys with properties tailored to meet increasingly stringent design requirements. At the macroscopic scale, little is known about the effect of solute chemistries on elastic properties of steels, such as Young's modulus, which is an important parameter for simulating springback in metal forming [16, 17, 18. Accurate data on solute-induced changes in the mechanical response of bcc $\mathrm{Fe}$ is therefore essential for designing new ferritic steels and multi-phase steels containing ferrite. However, modelling the complex interplay between the host crystal, solutes, microstructure, and other defects requires a multiscale approach with information from smaller length-scale simulations informing mesoscale models. Density functional theory (DFT) calculations provide accurate information on solute effects in metals, and have determined solute-dislocation interactions [19, 12, solute size misfits [19, and solute-dependent elastic stiffness coefficients [20, 21, 22, 8]. Changes in lattice

20 parameters and elastic stiffness coefficients can directly estimate solid-solution strengthening parameters [23, 24, 3, 19] and changes in ductility [25]. Mesoscale phase-field [26] and crystal plasticity models [27] of multi-phase steels are sensitive to the input lattice parameters and elastic stiffness coefficients of the different phases, and DFT data on the solute-dependence of these properties can enhance the predictive capabilities of these models [28].

In the present article, we consider the effects of the substitutional solutes $\mathrm{Al}, \mathrm{B}, \mathrm{Cu}, \mathrm{Mn}$, and $\mathrm{Si}$, and the interstitial solutes $\mathrm{C}$, and $\mathrm{N}$ on the lattice pa- 
rameter and elastic stiffness coefficients of bcc Fe. Available experimental data indicates how these solutes influence the ferrite phase [1, 2, 29. Aluminum is a deoxidizer that causes moderate age hardening by forming AlN precipitates in high-N steels. Silicon is a deoxidizer with strong solid solution strengthening and moderate age hardening, and also inhibits the formation of martensite. Boron is primarily added to increase hardenability. Copper and Mn increase strength, and improve hardenability and toughness through grain refinement.

35 Carbon is important in all steels since it is a highly effective hardener and strengthener, and forms carbides with other solutes. Nitrogen also increases strength by forming nitride precipitates with other solutes. Experimental studies 6, 3, 24, 4, measured the effects of several solutes including Al, Si, Mn, C, and $\mathrm{N}$ on the structural and mechanical properties of bcc Fe, and theoretical calculations determined the effects of $\mathrm{H}[7]$ and $\mathrm{B}[8]$ on the elastic stiffness coefficients. However, no comprehensive experiments or ab initio calculations have quantified the effects of $\mathrm{Al}, \mathrm{B}, \mathrm{Cu}, \mathrm{Mn}, \mathrm{Si}, \mathrm{C}$, and $\mathrm{N}$ on the lattice parameter and single-crystal elastic stiffness coefficients of bcc Fe. The lack of this information is a barrier to the broader development of crystal plasticity models of single- and multi-phase steels.

We apply DFT to compute the dependence of the bcc Fe lattice parameter and elastic stiffness coefficients on the concentration of $\mathrm{Al}, \mathrm{B}, \mathrm{Cu}, \mathrm{Mn}, \mathrm{Si}, \mathrm{C}$, and $\mathrm{N}$ solutes. We generalize the definition of solute size misfits [23] that quantify solute-induced volumetric expansion to more general solute-induced strains that can change the size and shape of a crystal. Solutes modify elastic stiffness coefficients through changes in crystal volume and chemical bonding [6, 30, 7]. Psiachos et al. 7] calculate the total and volumetric changes in the elastic stiffness coefficients of bcc Fe due to $\mathrm{H}$ solutes, and estimate the chemical contribution as the difference between the total and volumetric changes. We extend this approach by separately calculating the volumetric and chemical contributions to changes in the elastic stiffness coefficients. We show that the sum of these two contributions agrees with direct calculations of changes in the elastic stiffness coefficients that encompass both effects, verifying that the volumetric 
and chemical contributions are the dominant factors that determine how solutes modify elasticity and ultimately mechanical properties influenced by elastic response. The separate calculations of the volumetric and chemical contributions are more computationally efficient than the direct calculations, and also reveal the relative importance of solute-induced lattice strain and bonding effects for each solute.

The remainder of this paper is organized as follows. Section 2 discusses our methodology for computing solute effects on lattice parameters and elastic stiffness coefficients. We give the details of our DFT calculations for solutes in bcc Fe, and discuss the importance of choosing proper $k$-point meshes to ensure fast convergence of sensitive elastic stiffness coefficient derivatives used to determine the volumetric contributions to solute-induced changes in the elastic stiffness coefficients. The computed data and an assessment of its accuracy through comparisons with available DFT calculations and experimental data are discussed in Section 3. The major novel results in the paper are summarized in Section 4 .

\section{Methods}

We use DFT to compute the effects of the solutes $\mathrm{Al}, \mathrm{B}, \mathrm{Cu}, \mathrm{Si}, \mathrm{Mn}, \mathrm{C}$, and $\mathrm{N}$ on the lattice parameter and elastic stiffness coefficients $C_{i j}$ of bcc Fe at $0 \mathrm{~K}$. Experimental measurements show that effects of solutes and temperature on the lattice parameter [31] and elastic response [3] of bcc Fe are nearly independent. Our computed solute data can therefore be used in conjunction with separate data on temperature effects to give changes in these quantities as a function of solute concentration and temperature. We also demonstrate that the volumetric and chemical effects on the $C_{i j}$ can be computed independently, and that their sum agrees with solute-induced changes calculated directly using cubic supercells containing a solute with the lattice parameter relaxed to give zero hydrostatic stress. It is well known that $\mathrm{Al}, \mathrm{Cu}, \mathrm{Mn}$, and $\mathrm{Si}$ are substitutional solutes, and $\mathrm{C}$ and $\mathrm{N}$ are octahedral interstitial solutes in bcc Fe [2]. We treat B 
as a substitutional solute since experimental measurements [32, 33] supported by recent DFT calculations [34, 35, 8] show that at low temperature, B is more stable substitutionally than interstitially in bcc Fe. Experimental data 3 shows that the variation of the lattice parameter and polycrystalline elastic moduli of bcc Fe with solute concentration is approximately linear to $\sim 10$ at.\% for a large number of solutes. Solute concentrations relevant for advanced high strength steels 36 are generally below this level, so we expand the lattice parameter and $C_{i j}$ to first order in solute concentration and calculate the coefficients in the expansions. The similarity between the coefficients computed using $3 \times 3 \times 3$ and $4 \times 4 \times 4$ supercells verifies that higher-order contributions are small in the DFT calculations for the concentration range relevant for many technologically important steels. A similar methodology can be applied to steels with larger solute concentrations, but it may be necessary to include higher-order derivatives that capture the effects of solute-solute interactions. It may also be necessary to use supercells that better represent the atomic ordering of the alloy, e.g., special quasirandom structures [37, 38, can be used to model concentrated random alloys.

\subsection{Effect of solutes on the lattice parameter}

We define a strain misfit tensor $\epsilon_{i j}^{s}$ to quantify lattice distortions induced by a solute $s$ as the derivative of strain $e_{i j}$ with respect to solute concentration $c_{s}$ in the dilute limit. Physically, solutes induce stress $\sigma_{k l}$ in the host crystal causing the lattice to strain until the stress becomes zero. However, for computational efficiency we fix the lattice vectors of the simulation cells and compute the soluteinduced strain in the linear elastic limit from the induced stress and the elastic compliance tensor $S_{i j k l}$ as $e_{i j}=\sum_{k, l} S_{i j k l} \sigma_{k l}$. We introduce a single solute into a supercell and use DFT to compute $\sigma_{k l}$ after relaxing all of the ionic positions. We approximate $\epsilon_{i j}^{s}$ from the number of lattice sites $N$ in the supercell, the

elastic compliance tensor $S_{i j k l}^{0}$ of the pure crystal, and the computed stress $\sigma_{k l}$ 


$$
\epsilon_{i j}^{s}=\left.\frac{\partial e_{i j}}{\partial c_{s}}\right|_{c_{s}=0} \approx N \sum_{k, l} S_{i j k l}^{0} \sigma_{k l} .
$$

The expression on the right is a finite difference approximation for the derivative, where the factor of $N$ arises from $c_{s}=1 / N$. The strain misfit tensor can be computed for any crystal structure, and captures the effects of general soluteinduced elastic stresses that can arise from multiple solutes in a computational supercell. Here we focus on individual substitutional and octahedral interstitial solutes in bcc Fe which do not induce shear stress.

Substitutional solutes maintain the cubic symmetry of the bcc lattice, whereas octahedral interstitial solutes induce tetragonal stress that will break cubic symmetry if the lattice is allowed to fully relax. This leads to different expressions for the strain misfit tensors. Substitutional solutes induce isotropic stress $\sigma_{i j}=\sigma_{11} \delta_{i j}$ in bcc Fe, and the resulting solute strain misfit tensor is

$$
\epsilon_{i j}^{s}=\left.\frac{1}{a_{0}} \frac{\partial a}{\partial c_{s}}\right|_{c_{s}=0} \delta_{i j} \approx N\left(S_{1111}^{0}+2 S_{1122}^{0}\right) \sigma_{11} \delta_{i j},
$$

where $a_{0}$ is the lattice parameter of pure $\mathrm{Fe}$ and $\delta_{i j}$ is the Kronecker delta. The tetragonal stress induced by octahedral interstitial solutes in bcc Fe will change the lattice parameter along one crystal axis differently than along the other two perpendicular crystal axes if the lattice is fully relaxed. The resulting body-centered tetragonal (bct) structure would have lattice parameter $a_{1}$ along two crystal axes and a different lattice parameter $a_{3}$ along the third crystal axis. A [001]-oriented octahedral interstitial solute induces stress $\sigma_{i j}=\sigma_{11} \delta_{i j}\left(\delta_{i 1}+\right.$ $\left.\delta_{i 2}\right)+\sigma_{33} \delta_{i j} \delta_{i 3}$, and the resulting solute strain misfit tensor is

$$
\begin{aligned}
\epsilon_{i j}^{s} \approx N\left[\left(S_{1111}^{0}+S_{1122}^{0}\right) \sigma_{11}+S_{1122}^{0} \sigma_{33}\right] & \delta_{i j}\left(\delta_{i 1}+\delta_{i 2}\right) \\
& +N\left(2 S_{1122}^{0} \sigma_{11}+S_{1111}^{0} \sigma_{33}\right) \delta_{i j} \delta_{i 3} .
\end{aligned}
$$

In this case, $\epsilon_{11}^{s}=\epsilon_{22}^{s}=\left(1 / a_{0}\right)\left(\partial a_{1} / \partial c_{s}\right)_{c_{s}=0}$ and $\epsilon_{33}^{s}=\left(1 / a_{0}\right)\left(\partial a_{3} / \partial c_{s}\right)_{c_{s}=0}$. The strain misfit tensors for octahedral interstitial solutes in the [100]- and [010]- 
orientations are obtained by appropriate permutations of the tensor indices. Our computational supercells contain a single interstitial solute with a specific orientation that will transform the bcc lattice to a particular bct variant if the structure is allowed to fully relax. However, this study focuses on solute effects on the bcc ferrite phase of steels that contains many interstitials in each possible orientation causing isotropic expansion. We model this by constraining the supercells to be cubic, and determine isotropic changes in the lattice parameter from average cubic strain misfits $\overline{\epsilon^{s}}=\left(\epsilon_{11}^{s}+\epsilon_{22}^{s}+\epsilon_{33}^{s}\right) / 3$. The average strain misfit for substitutional solutes equals the diagonal tensor components $\overline{\epsilon^{s}}=\epsilon_{i i}^{s}$. The lattice parameter depends linearly on solute concentrations $c_{s}$ in the dilute limit with linear coefficients $a_{0} \bar{\epsilon}^{s}$ :

$$
a\left(\left\{c_{s}\right\}\right) \approx a_{0}+\left.\sum_{s} \frac{\partial a}{\partial c_{s}}\right|_{c_{s}=0} c_{s}=a_{0}\left[1+\sum_{s} \overline{\epsilon^{s}} c_{s}\right] .
$$

\subsection{Effects of solutes on the elastic stiffness coefficients}

We determine changes in the second-order elastic stiffness coefficients $C_{\alpha \beta \gamma \delta}$ due to solute-induced strain and changes in chemical bonding through separate calculations. In the rest of the discussion we use contracted Voigt notation [39] for the elastic stiffness coefficients, where the index pairs $\alpha \beta$ and $\gamma \delta$ are replaced by single indices $i$ and $j: C_{\alpha \beta \gamma \delta} \rightarrow C_{i j}$. We define the volumetric effect on $C_{i j}$ as the product of the pure $\mathrm{Fe} C_{i j}$ derivative with respect to lattice parameter $a$ and the derivative of $a$ with respect to solute concentration $c_{s}$ determined by $\overline{\epsilon^{s}}$ :

$$
\left(\frac{\partial C_{i j}}{\partial c_{s}}\right)_{\mathrm{vol}}=\left.\left.\left.\frac{\partial C_{i j}^{0}}{\partial a}\right|_{a=a_{0}} \cdot \frac{\partial a}{\partial c_{s}}\right|_{c_{s}=0} \approx \frac{\partial C_{i j}^{0}}{\partial a}\right|_{a=a_{0}} \cdot a_{0} \overline{\epsilon^{s}},
$$

where $C_{i j}^{0}$ are the elastic stiffness coefficients of pure Fe. We define the chemical effect on $C_{i j}$ as the derivative of $C_{i j}$ with respect to $c_{s}$ with $a$ constrained to equal the pure Fe lattice parameter $a_{0}$ :

$$
\left(\frac{\partial C_{i j}}{\partial c_{s}}\right)_{\text {chem }}=\left.\frac{\partial C_{i j}^{s}}{\partial c_{s}}\right|_{\substack{a=a_{0} \\ c_{s}=0}}
$$


where $C_{i j}^{s}$ are the elastic stiffness coefficients of Fe containing a solute. The total derivative of an elastic stiffness coefficient $\left(\partial C_{i j} / \partial c_{s}\right)_{\text {tot }}$ is the sum of the volumetric and chemical contributions,

$$
\left(\frac{\partial C_{i j}}{\partial c_{s}}\right)_{\mathrm{tot}}=\left(\frac{\partial C_{i j}}{\partial c_{s}}\right)_{\mathrm{vol}}+\left(\frac{\partial C_{i j}}{\partial c_{s}}\right)_{\mathrm{chem}} .
$$

The $C_{i j}$ depend linearly on solute concentrations in the dilute limit,

$$
C_{i j}\left(\left\{c_{s}\right\}\right) \approx C_{i j}^{0}+\sum_{s}\left(\frac{\partial C_{i j}}{\partial c_{s}}\right)_{\text {tot }} c_{s} .
$$

165

the volumetric effects, and we calculate the $C_{i j}$ in each case using stress-strain calculations rather than applying stress corrections to energy-strain calculations 40, 41, 42.

We also compute the $C_{i j}$ derivatives directly as

$$
\left(\frac{\partial C_{i j}}{\partial c_{s}}\right)_{\operatorname{dir}}=\left.\frac{\partial C_{i j}^{s}}{\partial c_{s}}\right|_{\substack{a=a_{\mathrm{eq}} \\ c_{s}=0}},
$$

where $a_{\mathrm{eq}}$ is the equilibrium zero-pressure lattice parameter for a cubic supercell containing a single solute. The direct calculations in Eqn. 9 validate the accuracy of Eqn. 7 since the direct supercells contain a solute and have relaxed lattice parameters, thereby including both the volumetric and chemical effects. However, computing the separate volumetric and chemical terms in Eqn. 7 is computationally more efficient since the $C_{i j}^{0}$ derivatives with respect to lattice parameter in the volumetric term are computed using one-atom primitive cells, and only atomic relaxations are required for computing the chemical terms. Furthermore, the separate calculations reveal the relative importance of the two different contributions in changing the anisotropic elastic response of the crystal lattice.

The tetragonal stress induced by octahedral interstitial solutes breaks the cubic symmetry of the $C_{i j}$ tensor even though the supercells are constrained to be cubic. We therefore average appropriate tensor elements [7] to obtain effective 
cubic $C_{i j}$ for bcc Fe containing $\mathrm{C}$ or N. For example, a [001]-oriented interstitial

results in $C_{11}=C_{22} \neq C_{33}, C_{13}=C_{23} \neq C_{12}$, and $C_{44}=C_{55} \neq C_{66}$. The average cubic $C_{i j}$ computed from the $C_{i j}$ of bcc Fe containing a [001]-oriented octahedral interstitial are therefore $\overline{C_{11}}=\left(2 C_{11}+C_{33}\right) / 3, \overline{C_{12}}=\left(C_{12}+2 C_{13}\right) / 3$, and $\overline{C_{44}}=\left(2 C_{44}+C_{66}\right) / 3$. We apply seven independent strains to compute the six independent elastic stiffness coefficients following the least-squares fitting procedure of Trinkle [43. Substitutional solutes induce isotropic stress which preserves the cubic symmetry of the elastic stiffness tensor, so no averaging is needed for these cases.

\subsection{DFT calculation details}

We use the plane-wave basis DFT code VASP [44] to compute $\epsilon_{i j}^{s}$ and soluteinduced changes in the $C_{i j}$ for bcc Fe. We compute the $C_{i j}$ from stress versus strain after relaxing all of the ion positions in the supercells. The Perdew-BurkeErnzerhof (PBE) generalized gradient approximation (GGA) functional [45] accounts for electron exchange and correlation energy, and a projector augmented wave (PAW) potential [46] with electronic configuration $[\mathrm{Ar}] 3 d^{7} 4 s^{1}$ generated by Kresse and Joubert [47] models the Fe nuclei and core electrons. The solutes $\mathrm{Al}$, $\mathrm{B}, \mathrm{Cu}, \mathrm{Mn}, \mathrm{Si}, \mathrm{C}$, and $\mathrm{N}$ are modelled using PAW potentials [47 with respective electronic configurations $[\mathrm{Ne}] 3 s^{2} 3 p^{1}$, [He] $2 s^{2} 2 p^{1},[\mathrm{Ar}] 3 d^{10} 4 s^{1},[\mathrm{Kr}] 3 d^{6} 4 s^{1}$, [Ne] $3 s^{2} 3 p^{2},[\mathrm{He}] 2 s^{2} 2 p^{2}$, and [He] $2 s^{2} 2 p^{3}$. The calculations require a plane-wave energy cutoff of $550 \mathrm{eV}$ to converge the energies to less than $1 \mathrm{meV} /$ atom. A conjugate gradient method relaxes the atoms until all of the atomic forces are less than $5 \mathrm{meV} / \AA$. The energy tolerance for the electronic self-consistency loop is $10^{-8} \mathrm{eV}$. All of the calculations are spin polarized to model the ferromagnetism of bcc Fe.

Figure 1 shows that the derivatives of the pure Fe bulk modulus $B_{0}$, shear modulus $C_{0}^{\prime}$, and $C_{i j}^{0}$ with respect to lattice parameter depend more strongly on $k$-point sampling than the energy per atom, lattice parameter $a_{0}, B_{0}, C_{0}^{\prime}$, and $C_{i j}^{0}$. The data in the plots is generated using the one-atom bcc primitive cell. We compute all the properties in the figure using standard $N_{k} \times N_{k} \times N_{k}$ Monkhorst- 
Pack (M-P) [48] $k$-point meshes, which are shifted off of the $\Gamma$-point for even numbers of mesh divisions. We also compute these properties using even M-P meshes that include the $\Gamma$-point. The energy per atom and lattice parameter oscillate around their converged values using $\Gamma$-centered and shifted meshes, but the magnitudes of the fluctuations are less than $1 \mathrm{meV}$ and $1 \mathrm{~m} \AA$. The fluctuations in the elastic moduli are relatively larger than the energy and lattice parameter fluctuations, but the values differ from the converged values by less than $2 \%$. In contrast, the fluctuations in the derivatives of $B_{0}, C_{0}^{\prime}$, and $C_{i j}^{0}$ with respect to lattice parameter are as large as $18 \%$. These derivatives are critically important since they determine $\left(\partial C_{i j} / \partial c_{s}\right)_{\mathrm{vol}}$ in Eqn. 5. so the standard M-P meshes lead to large errors in this quantity unless very dense meshes are used. 225 An improved $k$-point sampling method for the bcc lattice based on the simple cubic lattice [49] leads to faster convergence for all of these properties. The individual Monkhorst-Pack meshes form fcc meshes in reciprocal space, which combine to form a denser simple cubic mesh (black points in Fig. 1). The values we report for pure Fe properties are computed using the simple cubic $k$-point mesh generated by combining the $34 \times 34 \times 34 \Gamma$-centered and shifted meshes. Sampling the Brillouin zone with this simple cubic mesh is also equivalent to averaging the results from the two separate Monkhorst-Pack calculations since Brillouin zone integrations are approximated as weighted sums over the $k$-points. Our $2 \times 2 \times 2,3 \times 3 \times 3$, and $4 \times 4 \times 4$ cubic supercell calculations are converged 235 to a similar level of accuracy using $k$-point meshes generated by combining $12 \times 12 \times 12,8 \times 8 \times 8$, and $6 \times 6 \times 6 \Gamma$-point-centered and shifted MonkhorstPack meshes.

The data in Figure 1 is computed using order-one Methfessel-Paxton smearing [50] to ensure accurate forces and stresses for structural relaxations and 240 elastic stiffness coefficient calculations. We choose a smearing energy width of $0.2 \mathrm{eV}$ for our chosen $k$-point meshes to ensure close agreement between the electronic density of states (DOS) near the Fermi energy computed using the smearing method with the DOS computed using the linear tetrahedron method with Blöchl corrections [51]. We also investigated the sensitivity of the elastic 
stiffness coefficients and their derivatives to the smearing energy width. Varying the smearing width from $0.15 \mathrm{eV}$ to $0.25 \mathrm{eV}$ causes the elastic stiffness coefficients to change by less than $\sim 2 \%$. The elastic stiffness coefficients derivatives are more sensitive, varying from $\sim 2 \%$ for $\partial C_{12} / \partial a$ to $\sim 8 \%$ for $\partial C_{44} / \partial a$. Even though these errors are larger, the resulting variations in the solute-induced changes in the elastic stiffness coefficients as the smearing width changes from $0.15 \mathrm{eV}$ to $0.25 \mathrm{eV}$ are small. For example, the largest volumetric contributions are due to $\mathrm{C}$ and $\mathrm{N}$ since they induce the largest lattice strains. The changes in the elastic stiffness coefficients due to $\mathrm{C}$ and $\mathrm{N}$ in the $3 \times 3 \times 3$ supercell vary from $-23.4 \mathrm{GPa}$ to $-22.8 \mathrm{GPa}$ for $C_{11}$, from $-14.3 \mathrm{GPa}$ to $-14.0 \mathrm{GPa}$ for $C_{12}$, and from $-10.3 \mathrm{GPa}$ to $-9.8 \mathrm{GPa}$ for $C_{44}$. The variations for all the other solutes in the $3 \times 3 \times 3$ supercells, and all the solutes in the $4 \times 4 \times 4$ supercells are even smaller. We do not report values for the variations for the $2 \times 2 \times 2$ supercells since these calculations generally exhibit strong finite size effects.

\subsection{Computational workflow}

Figure 2 summarizes the workflow for computing $\epsilon_{i j}^{s}$ and the $C_{i j}$ derivatives with respect to $c_{s}$. The process begins with calculating $a_{0}, C_{i j}^{0}$, and the $C_{i j}^{0}$ derivatives with respect to lattice parameter for pure Fe. The $\epsilon_{i j}^{s}$ calculations follow, which require the pure Fe lattice parameter $a_{0}$ and elastic stiffness coefficients $C_{i j}^{0}$ as inputs. The final sets of calculations determine the $C_{i j}$ derivatives with respect to $c_{s}$. These calculations require inputs from the pure Fe and solute strain misfit calculations.

We start with optimizing the lattice parameter $a_{0}$ of pure Fe by computing the cell pressure for a small range of lattice parameter values centered around the experimental value. We then perform a quadratic fit to this data and determine the lattice parameter value where the fitted pressure is zero. The DFT-computed pressure for the optimal lattice parameter $a_{0}=2.832 \AA$ is less than 0.001 GPa. This lattice parameter is an input for computing the $C_{i j}^{0}$ of pure Fe, the solute strain misfits in Eqns. 2 and 3 , and the volumetric and chemical contributions to the $C_{i j}$ derivatives with respect to solute concentration in Eqns 5 and 6 . 
respectively.

The next set of calculations determine the single-crystal $C_{i j}^{0}$ of pure bcc Fe, which relate stress $\sigma_{i}$ to strain $e_{j}$ in the linear elastic limit as $\sigma_{i}=\sum_{j} C_{i j}^{0} e_{j}$. The computational cells in this set of calculations have the $a_{0}$ value computed in the previous step. We apply volumetric strains to compute the bulk modulus $B_{0}=\left(C_{11}^{0}+2 C_{12}^{0}\right) / 3$, volume-conserving orthorhombic strains 52 to compute the shear modulus $C_{0}^{\prime}=\left(C_{11}^{0}-C_{12}^{0}\right) / 2$, and volume-conserving monoclinic strains [52] to compute the shear modulus $C_{44}^{0}$. We use strain values of $\pm 0.25 \%$ and $\pm 0.5 \%$, which produce a linear stress-strain relation in each case. The slopes of the resulting stress-strain curves computed using a four-point central finite difference 55 give $B_{0}, C_{0}^{\prime}$, and $C_{44}^{0}$, and we solve for $C_{11}^{0}$ and $C_{12}^{0}$ from $B_{0}$ and $C_{0}^{\prime}$. The computed values are $C_{11}^{0}=278 \mathrm{GPa}, C_{12}^{0}=148 \mathrm{GPa}$, and $C_{44}^{0}=98 \mathrm{GPa}$. We determine the $C_{i j}^{0}$ derivatives with respect to lattice parameter by computing $C_{11}^{0}, C_{12}^{0}$, and $C_{44}^{0}$ for the lattice parameter values $a_{0} \pm 0.005$ $\AA$ and $a_{0} \pm 0.01 \AA$. We use the same four-point central difference formula to calculate the derivatives. The computed values are $\left.\left(\partial C_{11}^{0} / \partial a\right)\right|_{a \rightarrow a_{0}} ^{c_{s}=0}=-1323$ $\mathrm{GPa} / \AA,\left.\left(\partial C_{12}^{0} / \partial a\right)\right|_{a \rightarrow a_{0}} ^{c_{s}=0}=-828 \mathrm{GPa} / \AA$, and $\left.\left(\partial C_{44}^{0} / \partial a\right)\right|_{a \rightarrow a_{0}} ^{c_{s}=0}=-576 \mathrm{GPa} / \AA$. The $C_{i j}^{0}$ are inputs for the solute strain misfit calculations in Eqns. 2 and 3 , the chemical contributions to the $C_{i j}$ derivatives with respect to solute concentration in Eqn. 6, and the direct calculations of these $C_{i j}$ derivatives in Eqn. 9 295 (see Fig. 2). The $C_{i j}^{0}$ derivatives with respect to lattice parameter are inputs for the volumetric contributions to the $C_{i j}$ derivatives with respect to solute concentration in Eqn. 5 .

The solute strain misfit calculations follow next, which use $2 \times 2 \times 2$ (16 lattice sites), $3 \times 3 \times 3$ (54 lattice sites), and $4 \times 4 \times 4$ (128 lattice sites) cubic supercells with the pure Fe lattice parameter $a_{0}$ containing a single substitutional or octahedral interstitial solute atom. We constrain the supercell lattice vectors and relax the ionic positions. The stress $\sigma_{k l}$ in the supercells is non-zero after relaxing the atoms due to the constraint we impose on the lattice vectors. The number of lattice sites $N$, the stress $\sigma_{k l}$, and the elastic compliance coefficients $S_{i j k l}^{0}$ computed from the $C_{i j}^{0}$ determine the solute strain misfit tensors, 
using Eqn. 2 for substitutional solutes and Eqn 3 for interstitial solutes. The average $\overline{\epsilon^{s}}$ of the diagonal tensor values $\epsilon_{i i}^{s}$ for each solute is an input for calculating the volumetric contributions to the $C_{i j}$ derivatives with respect to solute concentration in Eqn. 5. The $\overline{\epsilon^{s}}$ values also provide initial estimates for the zero-pressure lattice parameter for each supercell with a solute. These initial lattice parameters and the atomic positions in the supercells are then optimized to give zero-pressure. The final geometries are strained to directly calculate the $C_{i j}$ derivatives with respect to solute concentration.

The chemical and volumetric contributions to the $C_{i j}$ derivatives with respect to solute concentration both require inputs from the calculations discussed above, but are independent of each other. We approximate the chemical contributions in Eqn. 6 using finite differences as

$$
\left(\frac{\partial C_{i j}}{\partial c_{s}}\right)_{\mathrm{chem}} \approx N\left[C_{i j}^{s}\left(a_{0}\right)-C_{i j}^{0}\right]
$$

where $C_{i j}^{s}\left(a_{0}\right)$ are elastic stiffness coefficients computed using cubic $2 \times 2 \times 2$, $3 \times 3 \times 3$, and $4 \times 4 \times 4$ supercells with the pure Fe lattice parameter $a_{0}$ that contain a single solute. We compute $C_{i j}^{s}\left(a_{0}\right)$ by applying the three independent strains used in the pure Fe calculations to the supercells containing substitutional solutes, and the seven independent strains discussed in the Methods section to the supercells containing interstitial solutes. The atoms are relaxed after applying strain in each case, and the elastic stiffness coefficients are extracted from the slopes of the DFT-computed stresses versus the applied strains. The volumetric contributions for each solute are computed from the pure Fe $C_{i j}^{0}$ derivatives with respect to lattice parameter, the pure Fe lattice parameter $a_{0}$, and the average solute strain misfit $\overline{\epsilon^{s}}$ using Eqn. 5 . The $C_{i j}$ derivatives from the chemical and volumetric calculations are input for the total contribution calculations in Eqn. 7 which sum these two independent contributions.

The final set of DFT calculations in Fig. 2 labeled "Direct calculations" compute the total $C_{i j}$ derivatives directly using cubic supercells that contain a single solute at their relaxed zero-pressure lattice parameter $a_{\text {eq }}$. This di- 
rect method encompasses both the chemical and volumetric contributions since Eqn. 9 using finite differences similar to Eqn. 10

$$
\left(\frac{\partial C_{i j}}{\partial c_{s}}\right)_{\mathrm{dir}} \approx N\left[C_{i j}^{s}\left(a_{\mathrm{eq}}\right)-C_{i j}^{0}\right]
$$

where $C_{i j}^{s}\left(a_{\mathrm{eq}}\right)$ is computed using cubic supercells that contain a single solute at their equilibrium zero-pressure lattice parameter $a_{\text {eq }}$. We use the same methodology as the chemical contributions to compute the elastic stiffness coefficients 


\section{Results and Discussion}

Figure 3 compares the strain misfit tensors for the substitutional and interstitial solutes and shows convergence with respect to supercell size. The strain misfit tensors are isotropic for substitutional solutes, with each diagonal element equal to the average value. The strain misfit tensors are anisotropic for interstitial solutes so we show the two independent components along with the average values. The strain misfits for substitutional $\mathrm{Al}, \mathrm{Cu}$, and $\mathrm{Mn}$ have similar positive values for the two largest supercell sizes, showing that these solutes cause the lattice to expand by similar amounts. Boron has a larger negative strain misfit reflecting its small ionic radius, which causes a larger lattice contraction. The Si strain misfit is nearly an order of magnitude smaller than the strain misfits of the other substitutional solutes, showing that Si has a smaller effect on the lattice parameter. The interstitial solutes have large positive strain misfits along the octahedral orientation direction and small negative strain misfits in the perpendicular directions, as discussed in Subsection 2.1. Note that the interstitial strain misfits are at least an order of magnitude larger than the substitutional strain misfits. This is due to large stress induced in the lattice by the close separation between the interstitial atom and its nearest Fe neighbors along the octahedral orientation direction. We compare our computed $\mathrm{Al}, \mathrm{Mn}$, Si, C, and N values to experimental data in the Data Validation section.

The strain misfits are inputs for Eqn. 4 which gives the lattice parameter as a function of solute concentration, and also determine the volumetric contributions to the elastic stiffness coefficient derivatives with respect to solute concentration in Eqn. 5. The concentration-dependent lattice parameter can be used directly as input for mesoscale models, and the solute strain misfits are generally a good indicator of the solid-solution strengthening of each solute [3]. Based on the strain misfit values, interstitial $\mathrm{C}$ and $\mathrm{N}$ should produce the largest increase in strength since they have the largest strain misfits followed by B. The solutes $\mathrm{Al}, \mathrm{Cu}$, and $\mathrm{Mn}$ should produce the next highest increase in strength, while the low value of the Si strain misfit indicates that it should have a small 
effect. The computed Si strain misfit agrees well with experimental data (see the Data Validation section), but $\mathrm{Si}$ is known to have a high strengthening potency in bcc Fe [24, 3] which has been attributed to strong elastic interactions between Si solutes and screw dislocations [24. The computed values vary between the three different supercells sizes, with the $2 \times 2 \times 2$ calculations subject to the largest finite size effects due to the small supercell size. The small variation between the $3 \times 3 \times 3$ and $4 \times 4 \times 4$ values are due to competing sources of error. The solute concentration that results from the $3 \times 3 \times 3$ supercell size $(1.85$ at.\%) is further from the dilute limit than the concentration from the $4 \times 4 \times 4$ supercell size (0.78 at.\%), and the $3 \times 3 \times 3$ supercell results are subject to larger finite size effects than the $4 \times 4 \times 4$ supercell results. The $4 \times 4 \times 4$ supercells are closer to the dilute limit, but the effects of solutes are smaller and the results are more sensitive to numerical errors. To help offset these competing errors we perform linear fits to the lattice parameter changes due to each solute in the $3 \times 3 \times 3$ and $4 \times 4 \times 4$ supercells, constrained to give the pure Fe lattice parameter $a_{0}$ for zero solute concentration. Table 1 lists the lattice parameter derivatives with respect to solute concentration which are slopes of the linear fits.

Figure 4 compares the volumetric and chemical contributions to the $C_{i j}$ derivatives with respect to solute concentration (Eqns. 5 and 6) and shows that sums of these terms (Eqn. 7) agree well with the direct calculations (Eqn. 9). The bars representing $\left(\partial C_{i j} / \partial c_{s}\right)_{\text {vol }}$ and $\left(\partial C_{i j} / \partial c_{s}\right)_{\text {chem }}$ are stacked on each other when these terms have the same sign so that the total length of both bars represents the sum of the two terms. If the terms have opposite signs, the larger of the two bars is shifted vertically from the axis so that the bars overlap to emphasize the partial cancellation between $\left(\partial C_{i j} / \partial c_{s}\right)_{\text {vol }}$ and $\left(\partial C_{i j} / \partial c_{s}\right)_{\text {chem }}$. For example, the bars corresponding to $\left(\partial C_{11} / \partial c_{\mathrm{B}}\right)_{\text {chem }}$ for each supercell size with B (medium red, blue, and orange) have been shifted upwards out of the negative range by an amount equal to that of the volumetric contributions ${ }_{420}$ (light red, blue, and orange) which are entirely in the positive range. The agreement between the sum of chemical and volumetric contributions with the 
Table 1: Derivatives of the lattice parameter $a$, single-crystal $C_{i j}, \mathrm{VRH}$ average polycrystalline Young's modulus $E_{\mathrm{VRH}}$ and shear modulus $G_{\mathrm{VRH}}$, and Pugh ratio $B_{\mathrm{VRH}} / G_{\mathrm{VRH}}$ with respect to solute concentration $c_{s}$. The values for the $a$ and $C_{i j}$ derivatives are the slopes of linear fits to the data from the $3 \times 3 \times 3$ and $4 \times 4 \times 4$ supercells. The values for the $E_{\mathrm{VRH}}, G_{\mathrm{VRH}}$, and $B_{\mathrm{VRH}} / G_{\mathrm{VRH}}$ derivatives are computed using Eqns. A.15 A.17 in the Appendix. The lattice parameter and $C_{i j}$ as a function of solute concentration are $a\left(\left\{c_{s}\right\}\right)=a_{0}+\sum_{c_{s}}\left(\partial a / \partial c_{s}\right)_{c_{s}=0} \cdot c_{s}$ and $C_{i j}\left(\left\{c_{s}\right\}\right)=C_{i j}^{0}+\sum_{c_{s}}\left(\partial C_{i j} / \partial c_{s}\right)_{\text {tot }} \cdot c_{s}$. Similar expressions give the solute dependence of $E_{\mathrm{VRH}}, G_{\mathrm{VRH}}$, and $B_{\mathrm{VRH}} / G_{\mathrm{VRH}}$. The lattice parameter derivatives have units of $\mathrm{m} \AA /$ at. $\%$ and the $C_{i j}, E_{\mathrm{VRH}}$, and $G_{\mathrm{VRH}}$ derivatives have units of $\mathrm{GPa} /$ at.\%. The $B_{\mathrm{VRH}} / G_{\mathrm{VRH}}$ derivatives have units of $1 /$ at. $\%$.

\begin{tabular}{cccccccc}
\hline Solute $s$ & $\mathrm{Al}$ & $\mathrm{B}$ & $\mathrm{Cu}$ & $\mathrm{Mn}$ & $\mathrm{Si}$ & $\mathrm{C}$ & $\mathrm{N}$ \\
\hline$\left.\frac{\partial a}{\partial c_{s}}\right|_{c_{s}=0}$ & 1.66 & -2.96 & 1.86 & 2.04 & -0.29 & 8.97 & 9.27 \\
$\left(\frac{\partial C_{11}}{\partial c_{s}}\right)_{\text {tot }}$ & -12.62 & -10.98 & -11.06 & -8.40 & -9.55 & -5.18 & -5.93 \\
$\left(\frac{\partial C_{12}}{\partial c_{s}}\right)_{\text {tot }}$ & -7.10 & -4.21 & -3.95 & -5.26 & -4.77 & -2.66 & -3.95 \\
$\left(\frac{\partial C_{44}}{\partial c_{s}}\right)_{\text {tot }}$ & 1.18 & -1.33 & -1.52 & 0.57 & 2.04 & -2.56 & -2.32 \\
$\left.\frac{\partial E_{\mathrm{VRH}}}{\partial c_{s}}\right|_{c_{s}=0}$ & -3.18 & -6.47 & -6.86 & -2.10 & -1.37 & -4.96 & -4.52 \\
$\left.\frac{\partial G_{\mathrm{VRH}}}{\partial c_{s}}\right|_{c_{s}=0}$ & -0.83 & -2.42 & -2.60 & -0.52 & -0.20 & -1.95 & -1.68 \\
$\frac{\partial}{\partial c_{s}}\left(\left.\frac{B \mathrm{VRH}}{G_{\mathrm{VRH}}}\right|_{c_{s}=0}\right.$ & -0.085 & -0.011 & -0.004 & -0.062 & -0.071 & 0.012 & -0.009 \\
\hline
\end{tabular}


direct calculations verifies that the separation of the chemical and volumetric contributions accounts for all of the essential physics that determines how solutes change the elasticity of bcc Fe. The chemical contributions are negative for most of the substitutional solutes, and larger than the volumetric contributions in most of the cases. All of the substitutional solutes cause $C_{11}$ and $C_{12}$ to decrease, which shows that these solutes cause a local weakening of bonds around the solute atom. Boron and $\mathrm{Cu}$ cause $C_{44}$ to decrease, whereas $\mathrm{Al}, \mathrm{Mn}$, and Si cause $C_{44}$ to increase. The interstitial solutes have large positive chemical contributions to the three $C_{i j}$ derivatives, since the interstitial atoms stiffen bonds by pushing neighboring Fe atoms closer together than in bulk. The large negative volumetric contributions of the interstitials reflects their large strain misfits. The two contributions partially cancel resulting in total values with magnitudes smaller or similar to the substitutional solute values for $C_{11}$ and $C_{12}$ derivatives, and larger for the $C_{44}$ derivatives. Interstitial $\mathrm{C}$ and $\mathrm{N}$ cause all the $C_{i j}$ to decrease with increasing solute concentration. The solute-dependent $C_{i j}$ determined by $\left(\partial C_{i j} / \partial c_{s}\right)_{\text {tot }}$ can be used directly as input for mesoscale models, and to predict solute-induced changes in the polycrystalline elastic moduli and Pugh ratio to estimate the influence of the solutes on ductility. Similar to the lattice parameter case, we perform linear fits to the total $C_{i j}$ data from the $3 \times 3 \times 3$ and $4 \times 4 \times 4$ supercell calculations to help offset competing finite-size and numerical errors. Table 1 lists the $C_{i j}$ derivatives with respect to solute concentration which are the slopes of the linear fits.

Table 1 lists the derivatives of the Voigt-Reuss-Hill (VRH) average polycrystalline Young's modulus $E_{\mathrm{VRH}}$, shear modulus $G_{\mathrm{VRH}}$, and Pugh ratio $B_{\mathrm{VRH}} / G_{\mathrm{VRH}}$ with respect to solute concentration, where $B_{\mathrm{VRH}}$ is the VRH bulk modulus. The derivatives are computed using Eqns. A.15 A.17 in the Appendix, which require the single-crystal $\partial C_{i j} / \partial c_{s}$, bulk modulus $B=\left(C_{11}+2 C_{12}\right) / 3$, shear modulus $C^{\prime}=\left(C_{11}-C_{12}\right) / 2$, and $C_{44}$ as input. The dilute-limit values listed 450 in the table are computed using the fitted $\left(\partial C_{i j} / \partial c_{s}\right)$ tot values in the table and the pure Fe bulk modulus $B_{0}$, shear modulus $C_{0}^{\prime}=\left(C_{11}^{0}-C_{12}^{0}\right) / 2$, and $C_{44}^{0}$. All of the solutes cause $E$ and $G$ to decrease, with $\mathrm{Cu}$ and B having the largest 
effect, followed by $\mathrm{C}$ and $\mathrm{N}$, and then $\mathrm{Al}, \mathrm{Mn}$, and $\mathrm{Si}$. The solutes $\mathrm{Cu}, \mathrm{B}, \mathrm{C}$, and $\mathrm{N}$ cause the smallest changes in the Pugh ratio, indicating that they have the smallest influence on ductility. The solutes $\mathrm{Al}, \mathrm{Mn}$, and $\mathrm{Si}$ lead to the largest decreases in the Pugh ratio, and corresponding decreases in ductility. We compare the computed dependence of $E_{\mathrm{VRH}}$ and $G_{\mathrm{VRH}}$ on $\mathrm{Mn}$ and C concentration to experimentally measured data in the Data Validation section.

\subsection{Data Validation}

We evaluate the accuracy of our computed pure Fe properties by comparing the results to previous plane-wave GGA DFT calculations [54, 55, 56, 57, 8, and available experimental data [58, 59, 60]. Our lattice parameter value $a_{0}=2.832$ $\AA$ closely matches the previous GGA results of 2.84-2.85 $\AA$, and agrees well with the experimental value of $2.866 \AA$ measured at $4.2 \mathrm{~K}$. Our elastic stiffness coefficient values $C_{11}^{0}=278 \mathrm{GPa}, C_{12}^{0}=148 \mathrm{GPa}$, and $C_{44}^{0}=98 \mathrm{GPa}$ are similar to the previous GGA results of $C_{11}^{0}=249-291 \mathrm{GPa}, C_{12}^{0}=139-151$ $\mathrm{GPa}$, and $C_{44}^{0}=70-110 \mathrm{GPa}$ and the experimental values $C_{11}^{0}=243.1 \mathrm{GPa}$, $C_{12}^{0}=138.1 \mathrm{GPa}$, and $C_{44}^{0}=121.9 \mathrm{GPa}$ measured at $4.2 \mathrm{~K}$. We convert our $C_{i j}^{0}$ derivatives with respect to lattice parameter into derivatives with respect to pressure for direct comparison with experimental data measured at $300 \mathrm{~K}$. The conversion factor is $-3 B_{0} / a_{0}$, where $B_{0}$ is the bulk modulus. Our computed values $\partial C_{11}^{0} / \partial P=6.54, \partial C_{12}^{0} / \partial P=4.09$, and $\partial C_{44}^{0} / \partial P=2.85$ closely match two sets of experimental values: $\partial C_{11}^{0} / \partial P=7.52,6.72, \partial C_{12}^{0} / \partial P=5.20,4.58$, and $\partial C_{44}^{0} / \partial P=2.66,2.59$. The good agreement of our calculations with exper475 iment shows that plane-wave GGA calculations accurately model the structural and elastic properties of bcc Fe.

We evaluate the accuracy of the computed strain misfits by comparing the corresponding predicted lattice parameters to the lattice parameters of cubic supercells containing the different solutes relaxed to yield zero pressure, and by direct comparison to available experimental data. The largest relative error between the predicted and directly computed lattice parameter values is $0.14 \%$ for $\mathrm{N}$ in the $2 \times 2 \times 2$ supercell. The errors for the $3 \times 3 \times 3$ and $4 \times 4 \times 4$ supercells 
are less than $0.02 \%$ for all the solutes. We have also removed the constraint of cubic symmetry for the supercells with [001]-octahedral C and fully optimized the geometry to verify the accuracy of Eqn. 3. The largest error of $1.56 \%$ occurs in the [100] and [010] directions for the $2 \times 2 \times 2$ supercell. The errors in all three directions for the $3 \times 3 \times 3$ and $4 \times 4 \times 4$ supercells are less than $0.06 \%$. Thus, the strain misfits accurately predict the relaxed DFT lattice parameters for all the solutes. Our computed solute strain misfits for $\mathrm{Al}$ of +0.056 for the $3 \times 3 \times 3$ supercell and +0.075 for the $4 \times 4 \times 4$ supercell agree well with the experimental value of +0.063 extracted from solid-solution strengthening measurements on Fe containing 5.85 at.\% $\mathrm{Al}$ at $300 \mathrm{~K}[24$. The $\mathrm{Si}$ strain misfit is nearly an order of magnitude smaller than the values for the other substitutional solutes. The $3 \times 3 \times 3$ supercell value is -0.012 and the $4 \times 4 \times 4$ supercell value is +0.003 . 495 This agrees with experimental data which show $\mathrm{Si}$ has a small effect on the bcc Fe lattice parameter, with measured misfit values ranging from -0.0084 for 1.38 at.\% Si 3] to -0.023 for 8.30 at.\% Si 24]. Our computed Mn value of +0.072 is about three times larger than the experimental value of 0.0234 for 2.97 at.\% Mn 3]. This may be due to inaccuracies within the DFT calculations for $\mathrm{Mn}$ in bcc Fe or clustering effects between Mn atoms that are absent in our supercell models, but a detailed study is needed to clarify these issues. Our average strain misfit values for $\mathrm{C}$ and $\mathrm{N}$ of +0.314 and +0.315 , respectively, are larger than any of our computed substitutional solute strain misfits. This is similar to the experimental trend, where the average strain misfit values for for the bct martensite phase containing $\mathrm{C}$ or $\mathrm{N}, 4$ are larger than the measured strain misfits for substitutional $\mathrm{Al}, \mathrm{Mn}$, and $\mathrm{Si}$. The differences between the experimental and computed strain misfit values cause the error in the predicted changes in lattice parameter to increase by less than $0.1 \%$ per $1 \%$ increase in solute concentration, even for $\mathrm{Mn}$ which shows the largest deviation from the experimental data.

The agreement between the sum of $\left(\partial C_{i j} / \partial c_{s}\right)_{\text {vol }}$ and $\left(\partial C_{i j} / \partial c_{s}\right)_{\text {chem }}$ with $\left(\partial C_{i j} / \partial c_{s}\right)_{\text {dir }}$ shown in Fig. 4 validates our computation approach, and com- 
parison with available experimental data assesses the accuracy of the computed data. To our knowledge there is no available experimental data on the solute dependence of the single-crystal $C_{i j}$ of bcc Fe, but the variation of the polycrystalline $E$ and $G$ with $\mathrm{Mn}$ and $\mathrm{C}$ concentration has been measured [6]. Figure 5 compares this data to the Voigt-Reuss-Hill polycrystalline averages computed from our single-crystal data. Both $\mathrm{Mn}$ and $\mathrm{C}$ cause $E$ and $G$ to decrease, with $\mathrm{C}$ having the largest effect. The similarity between the measured and computed data shows that our calculations predict the correct trends for the effects of $\mathrm{Mn}$ and $\mathrm{C}$ solutes on the polycrystalline moduli. The agreement between the magnitudes of the measured and computed data is also reasonable. The main source of error is the overestimation of the volumetric contributions to the $C_{i j}$ 525 derivatives, which is due to the computed strain misfit values for $\mathrm{Mn}$ and $\mathrm{C}$ being larger than the experimental values. The errors between the measured and computed polycrystalline moduli are substantially reduced if we use the experimental strain misfits to compute the volumetric contributions.

\section{Conclusions}

We present an efficient computational methodology to generate datasets on how solutes change the lattice parameters and elastic stiffness coefficients of single crystals. We provide a tensor generalization of the well-known concept of solute size misfit to describe lattice deformations due to anisotropic soluteinduced stresses. Solutes change the elastic stiffness coefficients $C_{i j}$ through volumetric and chemical effects, which are indicative of the amount of expansion or contraction caused by the insertion of a solute atom into the host lattice and changes in local chemical bonding in the lattice around the solute. We demonstrate that these two contributions can be computed separately at an accuracy comparable to more computationally expensive direct calculations. The computed solute dependence of the single-crystal $C_{i j}$ can be used to compute the solute dependence of the polycrystalline bulk modulus $B$, shear modulus $G$, and Young's modulus $E$ using standard averaging techniques, such as the 
Voigt-Reuss-Hill procedure which applies to statistically isotropic microstructures. We apply our methodology to substitutional $\mathrm{Al}, \mathrm{B}, \mathrm{Cu}, \mathrm{Mn}$, and $\mathrm{Si}$ solutes, and interstitial $\mathrm{C}$ and $\mathrm{N}$ solutes in bcc Fe, and show that the computed data agrees with available experimental data on solute effects in this phase of Fe. The computed data can be used to estimate how solutes effect mechanical properties like strength and ductility, and serve as input to increase the fidelity of larger length-scale mesoscopic models including crystal plasticity simulations. Additionally, the methods used to compute the effects of $\mathrm{Al}, \mathrm{B}, \mathrm{Cu}, \mathrm{Mn}, \mathrm{Si}, \mathrm{C}$, and $\mathrm{N}$ on bcc Fe can be applied to other solutes in other host lattices with different crystal symmetries. Thus, the framework presented can be applied with appropriate generalizations to computing solute effects on other Fe phases like austenite and martensite which can be incorporated into models of multiphase steels. Moreover, the methodology we present can also be used to compute changes in lattice parameters and elastic stiffness of insulating crystals due to charged point defects provided that the stress tensors are computed accurately using DFT. For charged defects, this requires properly accounting for the charge states as shown in Ref. 61 for size misfits in aliovalently doped $\mathrm{SrTiO}_{3}$. We provide the solute strain misfits and the single-crystal elastic stiffness coefficient derivatives with respect to solute concentration for the three different supercell sizes, along with the data for pure Fe in CSV files stored in the NIST dSpace repository (http://hdl.handle.net/11256/671). Reference [62] gives further information on the data stored in the repository and the format of the data files.

\section{Acknowledgements}

This material is based upon work supported by the Department of Energy National Energy Technology Laboratory under Award Number(s) DEEE0005976. This report was prepared as an account of work sponsored by an agency of the United States Government. Neither the United States Government nor any agency thereof, nor any of their employees, makes any warranty, express or implied, or assumes any legal liability or responsibility for the ac- 
curacy, completeness, or usefulness of any information, apparatus, product, or process disclosed, or represents that its use would not infringe privately owned rights. Reference herein to any specific commercial product, process, or service by trade name, trademark, manufacturer, or otherwise does not necessarily constitute or imply its endorsement, recommendation, or favoring by the United States Government or any agency thereof. The views and opinions of authors expressed herein do not necessarily state or reflect those of the United States Government or any agency thereof. The research was performed using computational resources sponsored by the Department of Energy's Office of Energy Efficiency and Renewable Energy and located at the National Renewable Energy Laboratory. Additional computational resources were provided by the National Energy Research Scientific Computing Center and the Taub cluster maintained and operated by the Computational Science and Engineering Program at the University of Illinois.

\section{Appendix A. Polycrystalline elastic moduli}

Here we give the Voigt, Reuss, and Voigt-Reuss-Hill expressions for the bulk modulus $B$, shear modulus $G$, and Young's modulus $E$ of an isotropic polycrystalline material, derived from the single-crystal elastic stiffness coefficients $C_{i j} 63$. The Voigt averages are derived under the assumption of uniform strain throughout the sample, and present an upper bound on $B$ and $G$. The Reuss averages are derived under the assumption of uniform stress, and present a lower bound on $B$ and $G$. The Voigt-Reuss-Hill values of these moduli are averages of the upper Voigt and lower Reuss bounds. The focus of this study is bcc Fe, so we only give expression that are valid for cubic crystals. Experiments usually measure $G$ and $E$, and derive $B$ values from the relation $B=G E /(9 G-3 E)$ (see, e.g, Ref. [6]). The Voigt (V) averages for the moduli are

$$
B_{\mathrm{V}}=\frac{C_{11}+2 C_{12}}{3}
$$




$$
\begin{gathered}
G_{\mathrm{V}}=\frac{C_{11}-C_{12}+3 C_{44}}{5}, \\
E_{\mathrm{V}}=\frac{\left(C_{11}+2 C_{12}\right)\left(C_{11}-C_{12}+3 C_{44}\right)}{2 C_{11}+3 C_{12}+C_{44}},
\end{gathered}
$$

where the $C_{i j}$ depend on solute concentration $c_{s}$. The Reuss (R) averages for the moduli are

$$
\begin{gathered}
B_{\mathrm{R}}=\frac{C_{11}+2 C_{12}}{3}, \\
G_{\mathrm{R}}=\frac{5\left(C_{11}-C_{12}\right) C_{44}}{3\left(C_{11}-C_{12}\right)+4 C_{44}}, \\
E_{\mathrm{R}}=\frac{5\left(C_{11}-C_{12}\right)\left(C_{11}+2 C_{12}\right) C_{44}}{C_{11}^{2}-C_{12}\left(2 C_{12}-C_{44}\right)+C_{11}\left(C_{12}+3 C_{44}\right)} .
\end{gathered}
$$

600

The Voigt-Reuss-Hill (VRH) $B_{\mathrm{VRH}}=\left(B_{\mathrm{V}}+B_{\mathrm{R}}\right) / 2$ and $G_{\mathrm{VRH}}=\left(G_{\mathrm{V}}+G_{\mathrm{R}}\right) / 2$ are

$$
\begin{gathered}
B_{\mathrm{VRH}}=\frac{C_{11}+2 C_{12}}{3}, \\
G_{\mathrm{VRH}}=\frac{3\left(C_{11}-C_{12}\right)^{2}+38\left(C_{11}-C_{12}\right) C_{44}+12 C_{44}{ }^{2}}{30\left(C_{11}-C_{12}\right)+40 C_{44}} .
\end{gathered}
$$

The VRH polycrystalline Young's modulus is most simply written as

$$
E_{\mathrm{VRH}}=\frac{9 B_{\mathrm{VRH}} G_{\mathrm{VRH}}}{3 B_{\mathrm{VRH}}+G_{\mathrm{VRH}}} .
$$

We note that $E_{\mathrm{VRH}}$ is not equivalent to $E_{\mathrm{avg}}=\left(E_{\mathrm{V}}+E_{\mathrm{R}}\right) / 2$, but they have similar values and similar derivatives for the solutes we consider in this study. The relative error between the dilute-limit values of the two derivatives range from $0.02 \%$ for $\mathrm{C}$ to $0.4 \%$ for $\mathrm{Si}$.

We present the derivatives of $G$ and $E$ with respect to solute concentration $c_{s}$ in terms of the $C_{i j}$ derivatives with respect to $c_{s}$. The coefficients in the expressions depend on $C_{11}, C_{12}$ and $C_{44}$, but the expressions are simpler when 
than this indicating brittle behavior. The derivative of the Pugh ratio with respect to $c_{s}$ can be used to estimate the effect solutes have on ductility. The derivatives of $G_{\mathrm{V}}$ and $E_{\mathrm{V}}$ with respect to $c_{s}$ are

$$
\begin{gathered}
\frac{\partial G_{\mathrm{V}}}{\partial c_{s}}=\frac{1}{5}\left(\frac{\partial C_{11}}{\partial c_{s}}-\frac{\partial C_{12}}{\partial c_{s}}\right)+\frac{3}{5} \frac{\partial C_{44}}{\partial c_{s}}, \\
\frac{\partial E_{\mathrm{V}}}{\partial c_{s}}=\left(15 B+2 C^{\prime}+3 C_{44}\right)^{-2}\left\{\left[135 B^{2}+3\left(2 C^{\prime}+3 C_{44}\right)^{2}\right] \frac{\partial C_{11}}{\partial c_{s}}\right. \\
\left.-\left[135 B^{2}-6\left(2 C^{\prime}+3 C_{44}\right)^{2}\right] \frac{\partial C_{12}}{\partial c_{s}}+405 B^{2} \frac{\partial C_{44}}{\partial c_{s}}\right\} .
\end{gathered}
$$

The derivatives of $G_{\mathrm{R}}$ and $E_{\mathrm{R}}$ with respect to $c_{s}$ are

$$
\begin{gathered}
\frac{\partial G_{\mathrm{R}}}{\partial c_{s}}=\left(3 C^{\prime}+2 C_{44}\right)^{-2}\left[5 C_{44}^{2}\left(\frac{\partial C_{11}}{\partial c_{s}}-\frac{\partial C_{12}}{\partial c_{s}}\right)+15 C^{\prime 2} \frac{\partial C_{44}}{\partial c_{s}}\right], \\
\frac{\partial E_{\mathrm{R}}}{\partial c_{s}}=\left(9 B C^{\prime}+6 B C_{44}+5 C^{\prime} C_{44}\right)^{-2}\left[15 C_{44}^{2}\left(9 B^{2}+5 C^{\prime 2}\right) \frac{\partial C_{11}}{\partial c_{s}}\right. \\
\left.-15 C_{44}^{2}\left(9 B^{2}-10 C^{\prime 2}\right) \frac{\partial C_{12}}{\partial c_{s}}+405 B^{2} C^{\prime 2} \frac{\partial C_{44}}{\partial c_{s}}\right] .
\end{gathered}
$$

${ }_{620}$ The derivatives of $B_{\mathrm{VRH}}, G_{\mathrm{VRH}}$, and $E_{\mathrm{VRH}}$ with respect to $c_{s}$ are

$$
\frac{\partial B_{\mathrm{VRH}}}{\partial c_{s}}=\frac{1}{3} \frac{\partial C_{11}}{\partial c_{s}}+\frac{2}{3} \frac{\partial C_{12}}{\partial c_{s}},
$$




$$
\begin{gathered}
\frac{\partial G_{\mathrm{VRH}}}{\partial c_{s}}=\left(3 C^{\prime}+2 C_{44}\right)^{-2}\left[\frac{9 C^{\prime 2}+12 C^{\prime} C_{44}+29 C_{44}^{2}}{10}\left(\frac{\partial C_{11}}{\partial c_{s}}-\frac{\partial C_{12}}{\partial c_{s}}\right)\right. \\
\left.\frac{51 C^{\prime 2}+18 C^{\prime} C_{44}+6 C_{44}{ }^{2}}{5} \frac{\partial C_{44}}{\partial c_{s}}\right], \quad \text { (A.15) } \\
\frac{\partial E_{\mathrm{VRH}}}{\partial c_{s}}=\left(3 B_{\mathrm{VRH}}+G_{\mathrm{VRH}}\right)^{-2}\left(9 G_{\mathrm{VRH}}{ }^{2} \frac{\partial B_{\mathrm{VRH}}}{\partial c_{s}}+27 B_{\mathrm{VRH}}{ }^{2} \frac{\partial G_{\mathrm{VRH}}}{\partial c_{s}}\right) .
\end{gathered}
$$

The derivative of the Pugh ratio $B_{\mathrm{VRH}} / G_{\mathrm{VRH}}$ with respect to $c_{s}$ is

$$
\frac{\partial}{\partial c_{s}}\left(\frac{B_{\mathrm{VRH}}}{G_{\mathrm{VRH}}}\right)=\frac{1}{G_{\mathrm{VRH}}} \frac{\partial B_{\mathrm{VRH}}}{\partial c_{s}}-\frac{B_{\mathrm{VRH}}}{G_{\mathrm{VRH}}{ }^{2}} \frac{\partial G_{\mathrm{VRH}}}{\partial c_{s}} .
$$

We give numerical values of Eqns. A.15 A.17 in Table 1 for the solutes Al, B, $\mathrm{Cu}, \mathrm{Mn}, \mathrm{Si}, \mathrm{C}$, and $\mathrm{N}$ in the dilute limit. We use the total derivative values $\left(\partial C_{i j} / \partial c_{s}\right)_{\text {tot }}$ in the table for $\left(\partial C_{i j} / \partial c_{s}\right)_{c_{s}=0}$ in Eqns. A.15 A.17. The singlecrystal $C_{i j}^{0}$ needed to compute the coefficients in Eqns. A.15 A.17 in the dilute limit are given in Subsection 2.4 .

\section{References}

[1] W. C. Leslie, The Physical Metallurgy of Steels, Techbooks, Herndon, 1991.

[2] H. Berns, W. Theisen, Ferrous Materials: Steel and Cast Iron, SpringerVerlag, Berlin, 2008.

[3] W. C. Leslie, Iron and its dilute substitutional solid solutions, Metal. Trans. 3 (1972) 5-26.

[4] L. Cheng, A. Böttger, T. H. de Keijser, E. J. Mittemeijer, Lattice parameters of iron-carbon and iron-nitrogen martensites and austenites, Scripta Metal. Mater. 24 (1990) 509-514. 
[5] C. M. Chu, H. Huang, P. W. Kao, D. Gan, Effect of alloying chemistry on the lattice constant of austenitic Fe-Mn-Al-C alloys, Scripta Metal. Mater. 30 (1994) 505-508.

[6] G. R. Speich, A. J. Schwoeble, W. C. Leslie, Elastic constants of binary iron-base alloys, Metall. Trans. 3 (1972) 2031-2037.

[7] D. Psiachos, T. Hammerschmidt, R. Drautz, Ab initio study of the modification of elastic properties of $\alpha$-iron by hydrostatic strain and by hydrogen interstitials, Acta Mater. 59 (2011) 4255-4263.

[8] A. F. Bialon, T. Hammerschmidt, R. Drautz, Ab initio study of boron in $\alpha$-iron: Migration barriers and interaction with point defects, Phys. Rev. B 87 (2013) 104109-1-104109-13.

[9] M. Souissi, H. Numakura, Elastic properties of FeC and FeN martensites, ISIJ Int. 55 (2015) 1512-1521.

[10] A. Abbasi, A. Dick, T. Hickel, J. Neugebauer, First-principles investigation of the effect of carbon on the stacking fault energy of FeC alloys, Acta. Mat. 59 (2011) 3041-3048.

[11] N. I. Medvedeva, M. S. Park, D. C. V. Aken, J. E. Medvedeva, Firstprinciples study of $\mathrm{Mn}, \mathrm{Al}$ and $\mathrm{C}$ distribution and their effect on stacking fault energies in fcc Fe, J. Alloys Comp. 582 (2014) 475-482.

[12] L. Ventelon, B. Luthi, E. Clouet, L. Proville, B. Legrand, D. Rodney, F. Willaime, Dislocation core reconstruction induced by carbon segregation in bcc iron, Phys. Rev. B 91 (2015) 220102-1(R)-220102-5(R).

[13] L. Samek, E. D. Moor, J. Penning, B. C. D. Cooman, Influence of alloying elements on the kinetics of strain-induced martensitic nucleation in lowalloy, multiphase high-strength steels, Metal. Mater. Trans. A 37 (2006) $109-124$. 
[14] E. Sak-Saracinco, H. M. Urbassek, Free energies of austenite and martensite fec alloys: an atomistic study, Phil. Mag. 94 (2013) 933-945.

[15] X. Zhang, T. Hickel, J. Rogal, S. Fähler, R. Drautz, J. Neugebauer, Structural transformations among austenite, ferrite and cementite in $\mathrm{Fe}-\mathrm{C}$ alloys: A unified theory based on ab initio simulations, Acta Mater. 99 (2015) 281289.

[16] B. Harris, The influence of some solutes on young's modulus of niobium, J. Less Common Met. 12 (1967) 247-250.

[17] W. Gan, S. S. Babu, N. Kapustka, R. H. Wagoner, Microstructural effects on the springback of advanced high-strength steel, Metall. Mater. Trans. A 37 (2006) 3221-3231.

[18] A. Andersson, Numerical and experimental evaluation of springback in advanced high strength steel, J. Mater. Eng. Perform. 16 (2007) 301-307.

[19] J. Yasi, L. G. H. Jr., D. R. Trinkle, First-principles data for solid-solution strengthening of magnesium: From geometry and chemistry to properties, Acta Mater. 58 (2010) 5704-5713.

[20] S. Ganeshan, S. L. Shang, Y. Wang, Z.-K. Liu, Effect of alloying elements on the elastic properties of $\mathrm{Mg}$ from first-principles calculations, Acta Mater. 57 (2009) 3876-3884.

[21] Z.-K. Liu, H. Zhang, S. Ganeshan, Y. Wang, S. N. Mathaudhu, Computational modeling of effects of alloying elements on elastic coefficients, Scripta Mater. 63 (2010) 686-691.

[22] M. de Jong, D. L. Olmstead, A. van de Walle, M. Asta, First-principles 685 study of the structural and elastic properties of rhenium-based transitionmetal alloys, Phys. Rev. B 86 (2012) 224101-1-224101-9.

[23] R. L. Fleisher, in The Strengthening of Metals, edited by D. Peckner, Reinhold, New York, 1964. 
[24] S. Takeuchi, Solid-solution strengthening in single crystals of iron alloys, J. Phys. Soc. Japan 27 (1969) 929-940.

[25] S. F. Pugh, Relations between the elastic moduli and the plastic properties of polycrystalline pure metals, Phil. Mag. 45 (1954) 823-843.

[26] H. K. Yeddu, A. Malik, J. Ågren, G. Amberg, A. Borgenstam, Threedimensional phase-field modeling of martensitic microstructure evolution in steels, Acta Mater. 60 (2012) 1538-1547.

[27] A. Srivastava, A. F. Bower, L. G. H. Jr., J. E. Carsley, L. Zhang, F. AbuFarha, A multiscale approach to modeling formability of dual-phase steels, Modelling Simul. Mater. Sci. Eng. 24 (2016) 025011-1-025011-30.

[28] F. Roters, P. Eisenlohr, L. Hantcherli, D. D. Tjahjanto, T. R. Bieler, D. Raabe, Overview of constitutive laws, kinematics, homogenization and multiscale methods in crystal plasticity finite-element modeling: Theory, experiments, applications, Acta Mater. 58 (2010) 1152-1211.

[29] R. E. Smallman, A. H. W. Ngan, Modern Physical Metallurgy, 8th edition, Butterworth-Heinemann, Waltham, 2014.

${ }_{705}$ [30] O. Buck, L. A. Ahlberg, L. J. Graham, G. A. Alers, C. A. Wert, K. C. Hsieh, Harmonic and anharmonic elastic properties of the $\alpha-\mathrm{Nb}-\mathrm{H}$ system, Phys Status Solidi (a) 55 (1979) 223-230.

[31] Y. S. Touloukian, R. K. Kirby, R. E. Taylor, P. D. Desai, Thermophysical Properties of Matter: Thermal Expansion - Metallic Elements and Alloys, Vol. 12, Plenum Press, 1975.

[32] P. E. Busby, C. Wells, Diffusion of boron in alpha-iron, J. Met. 6 (1954) 972-972.

[33] P. M. Strocchi, B. A. Melandri, A. Tamba, On the nature of boron solid solution in $\alpha$-iron, Nuovo Cimento B 51 (1967) 1-11. 
[42] X.-F. Li, G.-F. Ji, F. Zhao, X.-R. Chen, D. Alfè, First-principles calculations of elastic and electronic properties of $\mathrm{NbB}_{2}$ under pressure, J. Phys.: Condens. Matter 21 (2009) 025505-1-025505-7.

[43] D. R. Trinkle, Lattice and elastic constants of titanium-niobium monoborides containing aluminum and vanadium, Scripta Mat. 56 (2007) 273276. 
[44] G. Kresse, J. Furthmüller, Efficient iterative schemes for ab initio totalenergy calculations using a plane-wave basis set, Phys. Rev. B 54 (1996) 11169-11186.

[45] J. P. Perdew, K. Burke, M. Ernzerhof, Generalized gradient approximation made simple, Phys. Rev. Lett. 77 (1996) 3865-3868.

[46] P. E. Blöchl, Projector augmented-wave method, Phys. Rev. B 50 (1994) 17953-17979.

[47] G. Kresse, D. Joubert, From ultrasoft pseudopotentials to the projector augmented-wave method, Phys. Rev. B 59 (1999) 1758-1775.

[48] H. J. Monkhorst, J. D. Pack, Special points for Brillouin-zone integrations, Phys. Rev. B 13 (1976) 5188-5192.

[49] D. J. Singh, L. N. (eds.), Planewaves, Pseudopotentials, and the LAPW Method, 2nd edition, Springer, New York, 2006.

[50] M. Methfessel, A. T. Paxton, High-precision sampling for Brillouin-zone integration in metals, Phys. Rev. B 40 (1989) 3616-3621.

[51] P. E. Blöchl, O. Jepsen, O. K. Andersen, Improved tetrahedron method for Brillouin-zone integrations, Phys. Rev. B 49 (1994) 16223-16233.

[52] M. J. Mehl, B. M. Klein, D. A. Papaconstantopoulos, in Intermetallic Compounds: Principles and Practice, Volume I: Principles, edited by J. H. Westbrook and R. L. Fleischer, John Wiley and Sons, London, 1995.

[53] B. Fornberg, Generation of finite difference formulas on arbitrarily spaced grids, Math. Comp. 51 (1988) 699-706.

765 [54] G. Y. Guo, H. H. Wang, Gradient-corrected density functional calculation of elastic constants of $\mathrm{Fe}$, Co and $\mathrm{Ni}$ in bcc, fcc, and hcp structures, Chinese J. Phys. 38 (2000) 949-961. 
[55] S. L. Frederiksen, K. W. Jacobsen, Density functional theory studies of screw dislocation core structures in bcc metals, Phil. Mag. 83 (2003) 365375.

[56] K. J. Caspersen, A. Lew, M. Ortiz, E. A. Carter, Importance of shear in the bcc-to-hcp transformation in iron, Phys. Rev. Lett. 93 (2004) 115501$1-115501-4$.

[57] L. Ventelon, F. Willaime, Generalized stacking-faults and screw-dislocation core-structure in bcc iron: A comparison between ab initio calculations and empirical potentials, Phil. Mag. 90 (2009) 1063-1074.

[58] J. A. Rayne, B. S. Chandrasekhar, Elastic constants of iron from $4.2^{\circ} \mathrm{K}$ to $300^{\circ}$ K, Phys. Rev. 122 (1961) 1714-1716.

[59] C. A. Rotter, C. S. Smith, Ultrasonic equation of state of iron. i. low pressure, room temperature, J. Phys. Chem. Solids 27 (1966) 267-276.

[60] M. W. Guinan, D. N. Beshers, Pressure derivatives of the elastic constants of $\alpha$-iron to $10 \mathrm{Kbs,} \mathrm{J.} \mathrm{Phys.} \mathrm{Chem.} \mathrm{Solids} 29$ (1968) 541-549.

[61] H. Kim, D. R. Trinkle, Effect of charge on point defect size misfits from ab initio: Aliovalently doped $\mathrm{SrTiO}_{3}$, Comp. Mat. Sci. 119 (2016) 41-45.

[62] M. R. Fellinger, L. G. Hector, Jr., D. R. Trinkle, Data files for ab initio calculations of the lattice parameter and elastic stiffness coefficients of bcc Fe with solutes, Data in Brief submitted.

[63] G. Grimvall, Thermophysical Properties of Materials, 2nd edition, North Holland, 1999.

[64] G. Wang, S. Shönecker, S. Hertzman, Q.-M. Hu, B. Johansson, S. K. Kwon, L. Vitos, Ab initio prediction of the mechanical properties of alloys: The case of Ni/Mn-doped ferromagnetic Fe, Phys. Rev. B 91 (2015) 224203. 

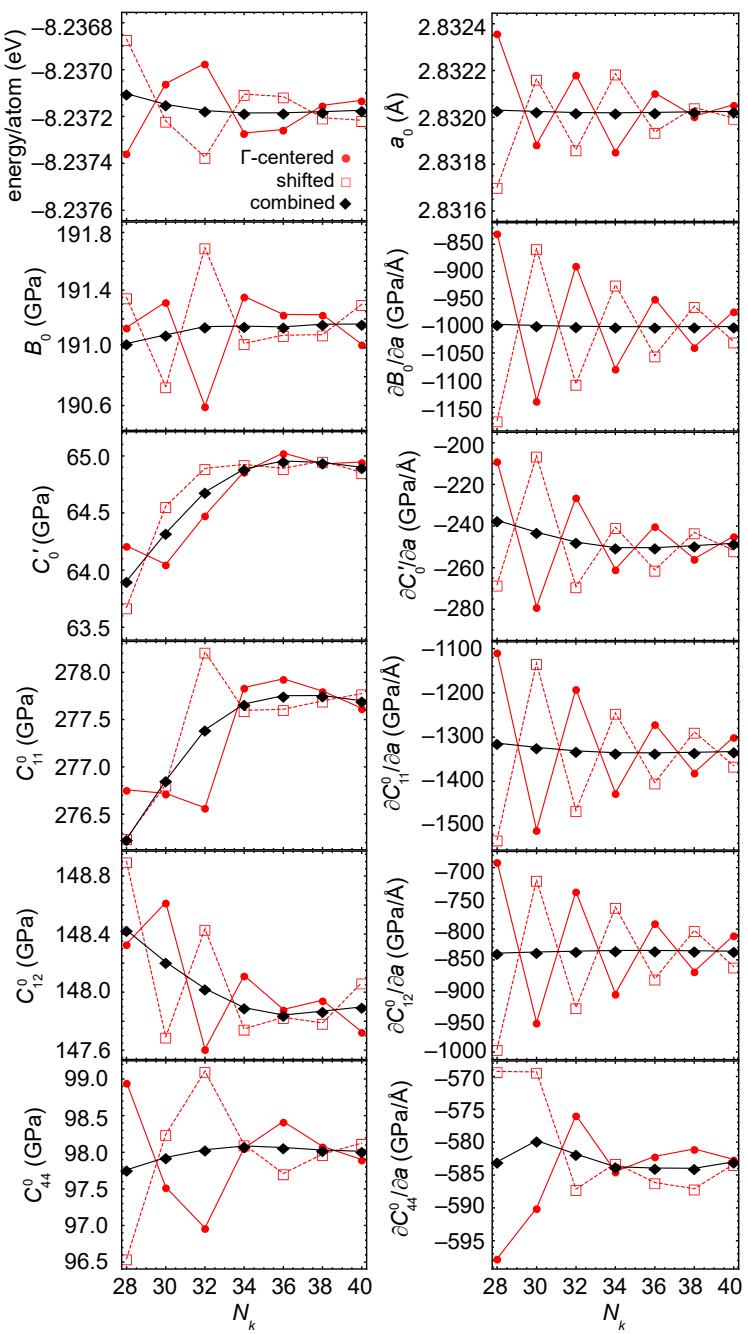

Figure 1: (color online). Convergence of pure bcc Fe properties with respect to $k$-point sampling. We compute the energy per atom, lattice parameter $a_{0}$, bulk modulus $B_{0}$, shear modulus $C_{0}^{\prime}$, and elastic stiffness coefficients $C_{i j}^{0}$, and the elastic moduli and stiffness coefficient derivatives with respect to $a$ using three different $k$-point meshes. We use $N_{k} \times N_{k} \times N_{k} \Gamma$ centered and shifted Monkhorst-Pack [48 meshes for the bcc lattice. These individual meshes are fcc meshes of points in $k$-space, which form a denser simple cubic mesh of points when combined together. The individual meshes lead to slower convergence with respect to $k$-points than the combined meshes for all the properties. This is especially pronounced for the elastic stiffness coefficient derivatives, which show large oscillations with respect to $k$-points for the individual Monkhorst-Pack meshes. 


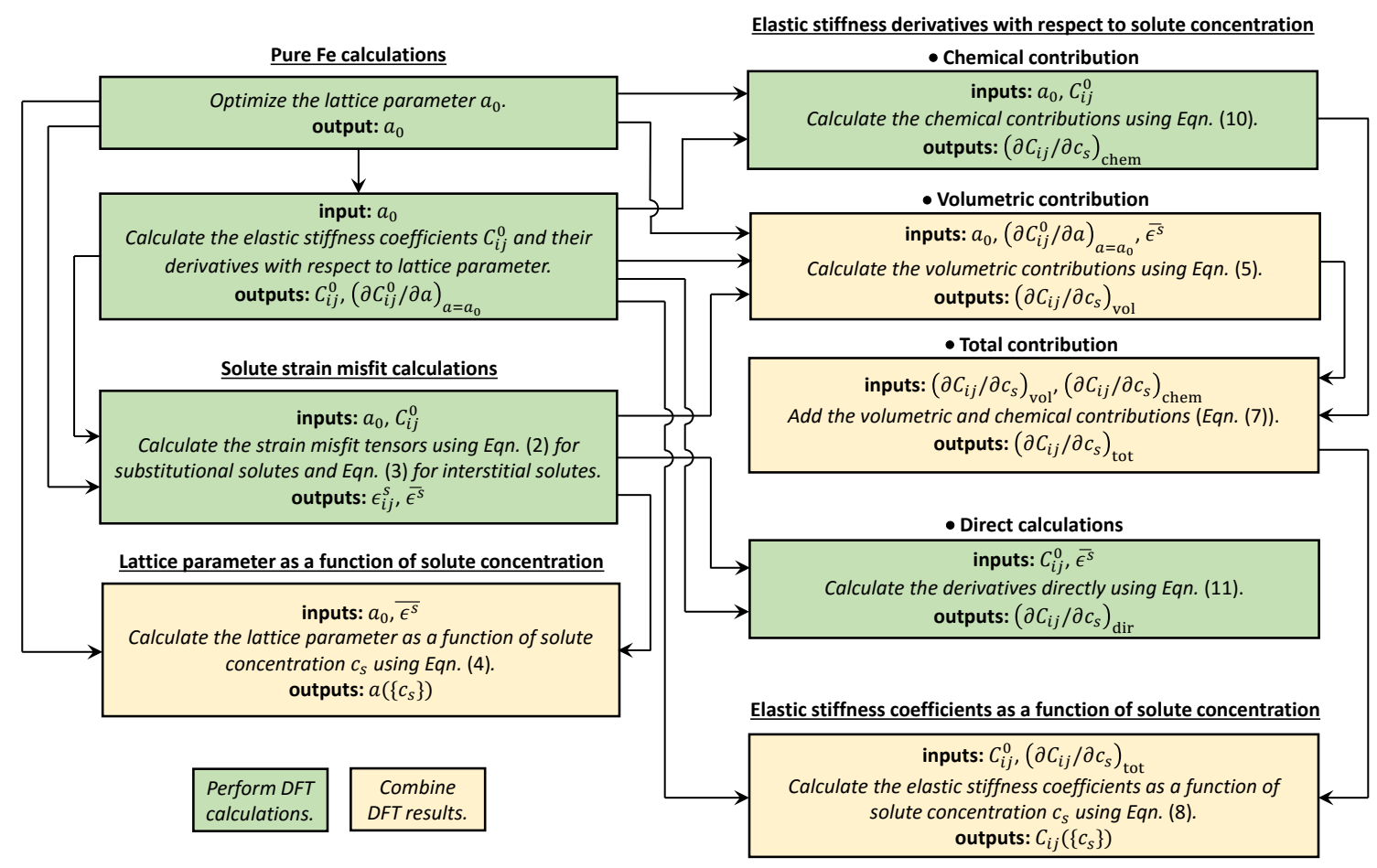

Figure 2: (color online). Workflow for computing solute strain misfits and elastic stiffness coefficient derivatives with respect to solute concentration. The arrows show which outputs from a given calculation are inputs to other calculations. The green boxes within each section represent sets of DFT calculations and post-processing of the DFT data. The yellow boxes represent steps in which no DFT calculations are performed, but use DFT results from prior steps in the workflow to calculate properties. 

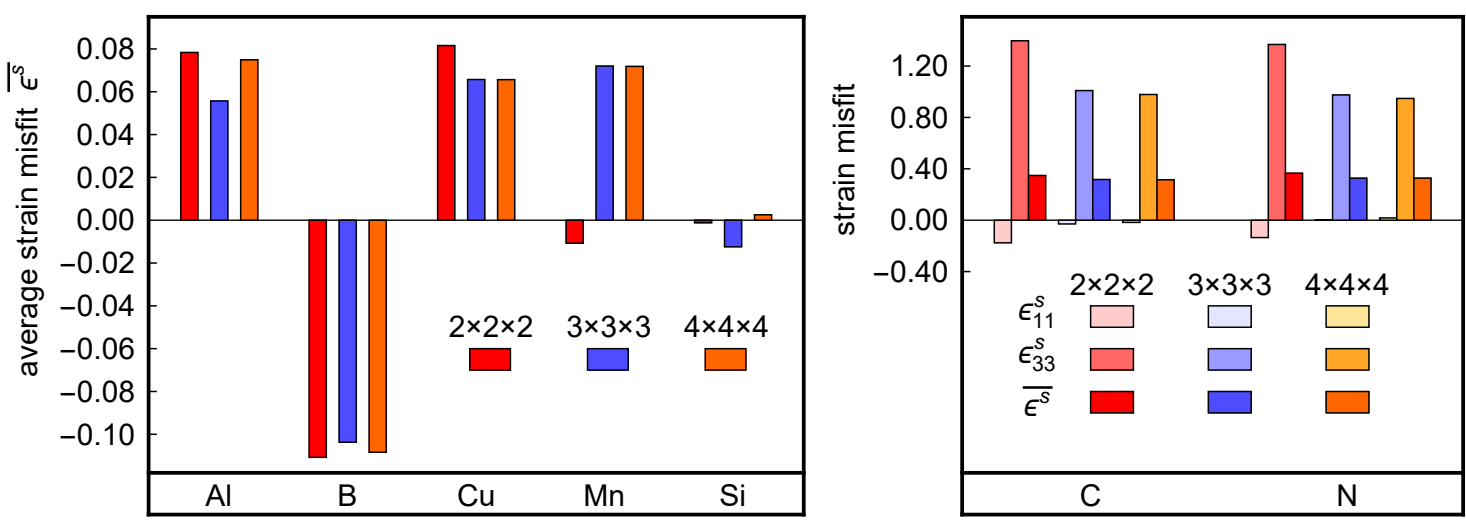

Figure 3: (color online). Strain misfit tensor values for substitutional (left) and octahedral interstitial (right) solutes. We compute the tensors using $2 \times 2 \times 2$ (16 lattice sites), $3 \times 3 \times 3$ (54 lattice sites), and $4 \times 4 \times 4$ (128 lattice sites) supercells to examine convergence with respect finite-size effects. The supercells contain a single solute which yields solute concentrations of $6.25,1.85$, and 0.78 at.\%, respectively. The misfit tensors for the substitutional solutes are isotropic so $\epsilon_{i i}^{s}=\overline{\epsilon^{s}}$, whereas the misfit tensors are anisotropic for the interstitial solutes. The strongest finite-size effects occur for $\mathrm{Mn}$, which has a small negative strain misfit value for the $2 \times 2 \times 2$ supercell and larger positive values for the $3 \times 3 \times 3$ and $4 \times 4 \times 4$ supercells. This behavior is reflected by the Mn magnetic moment which changes from +0.9 in the $2 \times 2 \times 2$ supercell to -2.0 in the larger supercells. The strain misfits for interstitial $\mathrm{C}$ and $\mathrm{N}$ are an order of magnitude larger than the substitutional strain misfits due to the large stress they induce in the bcc lattice. The data in the figure is for atomic concentration. For atomic percent, the values must be divided by 100 . 


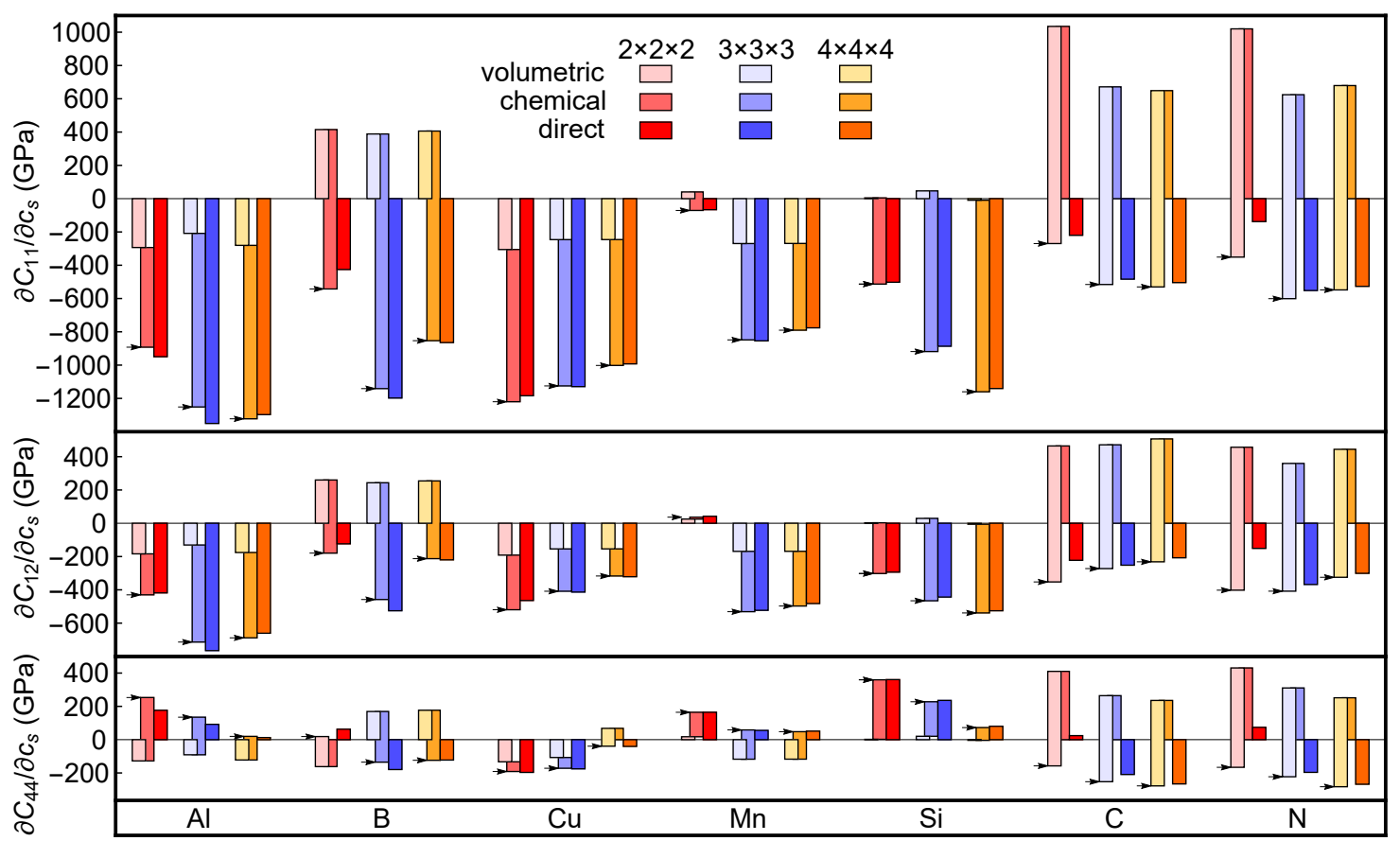

Figure 4: (color online). Single-crystal elastic stiffness coefficient derivatives with respect to solute concentration for $2 \times 2 \times 2$ (16 lattice sites), $3 \times 3 \times 3$ (54 lattice sites), and $4 \times$ $4 \times 4$ (128 lattice sites) supercells. The supercells contain a single solute which yields solute concentrations of $6.25,1.85$, and 0.78 at.\%, respectively. We compute volumetric and chemical contributions to the $C_{i j}$ derivatives separately and sum them to give total derivatives. We also compute the total derivatives directly using fully relaxed supercells containing solutes. Each supercell size for each solute has three bars in the figure: one for the volumetric effect, one for the chemical effect, and one for the direct calculations. The volumetric and chemical bars are stacked on each other when they have the same sign so that the total change is the combined length of both bars. When the terms have opposite signs, the larger bar is shifted vertically so that the bars overlap to demonstrate the partial cancellation of the two effects. The small arrows indicate the sums of the volumetric and chemical contributions, which agree well with the directly computed derivatives. The disagreement for the smallest supercell size in some cases indicates finite size effects. The data in the figure is for atomic concentration. For atomic percent, the values must be divided by 100 . 


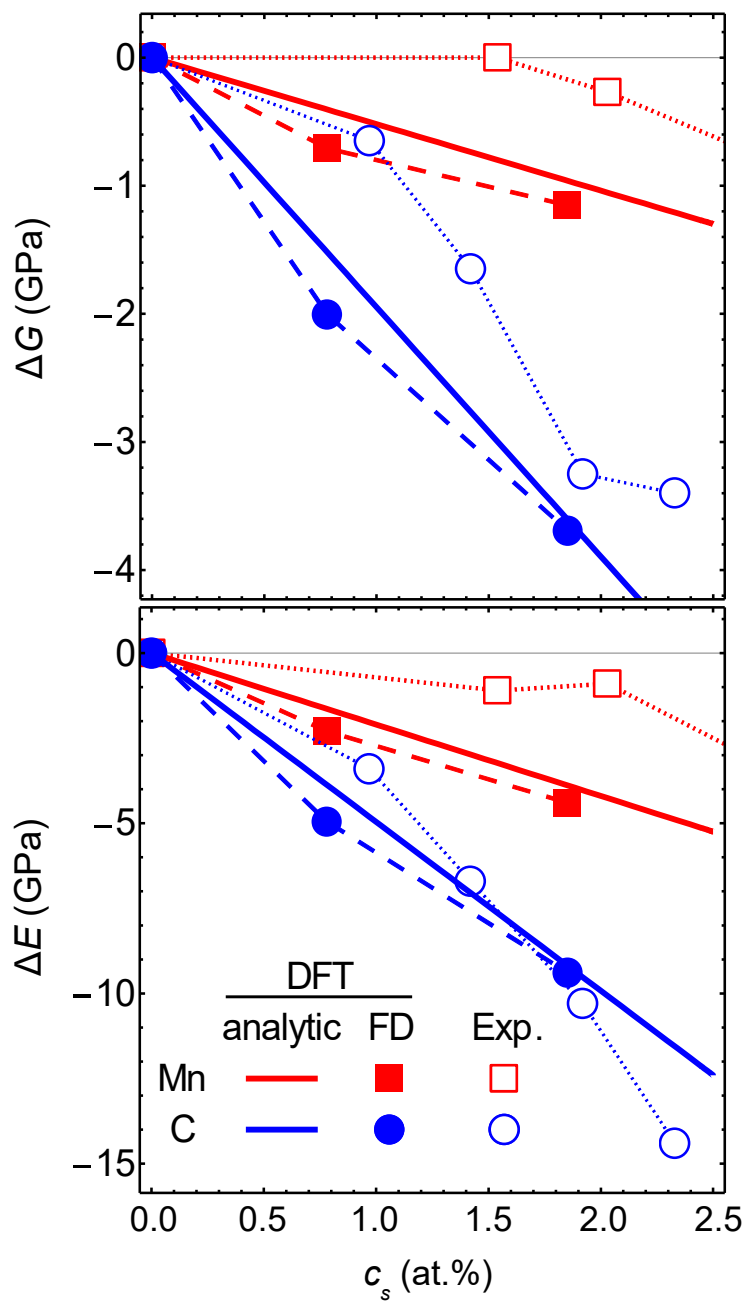

Figure 5: (color online). Computed changes in the Voigt-Reuss-Hill polycrystalline shear modulus $G$ and Young's modulus $E$ due to $\mathrm{Mn}$ and $\mathrm{C}$ solutes compared to experimental data. The DFT results labeled "analytic" are lines with slopes given by Eqn. A.15 and Eqn. A.16 in the Appendix, with numerical values given in Table 1 The DFT results labeled "FD" are finite difference changes in $G$ and $E$ computed from the single-crystal $C_{i j}$ from the $4 \times 4 \times 4$ and $3 \times 3 \times 3$ supercells using Eqn. A.8 and Eqn. A.9 These $C_{i j}$ values equal $C_{i j}^{0}+\left(\partial C_{i j} / \partial c_{s}\right)_{\text {tot }} \cdot c_{s}$, where we use the $\left(\partial C_{i j} / \partial c_{s}\right)_{\text {tot }}$ values computed for each supercell size. The $4 \times 4 \times 4$ supercells have $c_{s}=0.78$ at. $\%$ and the $3 \times 3 \times 3$ supercells have $c_{s}=1.85$ at.\%. The dashed lines connecting the FD points are guides for the eye. The variation in the FD results between the two supercell sizes is due to the small variations in the $C_{i j}$ computed using these different supercell sizes. The analytic results are derived from the fitted $C_{i j}$ derivative values in Table 1 which smooth out these variations. The DFT results agree with the trends in the experimental data from Speigh et al. [6] which show that these solutes cause both moduli to decrease, with $\mathrm{C}$ producing a larger effect on each modulus than $\mathrm{Mn}$. The dotted lines connecting the experimental data points are guides for the eye. 